\title{
The Diamond Integral on Time Scales*
}

\author{
Artur M. C. Brito da Cruz ${ }^{1,2}$ \\ artur.cruz@estsetubal.ips.pt \\ Natália Martins ${ }^{2}$ \\ natalia@ua.pt \\ Delfim F. M. Torres ${ }^{2}$
delfim@ua.pt \\ ${ }^{1}$ Escola Superior de Tecnologia de Setúbal, Estefanilha, 2910-761 Setúbal, Portugal \\ ${ }^{2}$ CIDMA-Center for Research and Development in Mathematics and Applications, \\ Department of Mathematics, University of Aveiro, 3810-193 Aveiro, Portugal
}

\begin{abstract}
We define a more general type of integral on time scales. The new diamond integral is a refined version of the diamond-alpha integral introduced in 2006 by Sheng et al. A mean value theorem for the diamond integral is proved, as well as versions of Holder's, Cauchy-Schwarz's and Minkowski's inequalities.
\end{abstract}

Keywords: diamond integral; time scales; integral inequalities.

2010 Mathematics Subject Classification: 26D15; $26 \mathrm{E} 70$.

\section{Introduction}

In 1988, Hilger introduced the theory of time scales in his dissertation. This intends to unify and extend existing continuous and discrete calculus into a uniformed theory [1, 2. Since then, the time scale theory advanced fast, as seen by the number of published works dedicated to the subject. Science Watch, from Thomson Reuters, considered in October 2007, and later in February 2011, the study of equations on time scales as an emerging research front in the field of mathematics with "potential applications in such areas as engineering, biology, economics and finance, physics, chemistry, social sciences, medical sciences, mathematics education, and others". For a good introduction to the theory of time scales, we refer the reader to the comprehensive books [3, 4 .

The delta/forward calculus and the nabla/backward calculus were the first approaches of the calculus on time scales. In 2006, a combined diamond- $\alpha$ dynamic derivative (resp. integral) was introduced by Sheng, Fadag, Henderson and Davis, as a linear combination of the delta and nabla dynamic derivatives (resp. integral) on time scales [5. Sheng et al. showed that those diamond- $\alpha$ derivatives offer more balanced approximations for computational applications. Having in mind that the classical symmetric derivative is a generalization of the classical derivative and that the classical symmetric difference quotient is generally a more accurate approximation for the derivative than the usual one-sided quotient, an important question consists to define a symmetric derivative on time scales, as a generalization of the diamond- $\alpha$ derivative [6]. On the other hand, the problem of determining a symmetric integral is a topic that captured the interest of many important mathematicians along the history, such as Denjoy, James, Kurzweil and Jarník. However, it is generally accepted that the integrals proposed only "invert approximately" the corresponding derivative [7. Despite this apparent limitation, these integrals have nice properties. For example, they are useful to solve the coefficient problem for trigonometric series when the conventional integral fails to exist [7. Our goal here is to define the diamond integral as an "approximate" symmetric integral on time scales (see Section 3).

${ }^{*}$ This is a preprint of a paper whose final and definite form will appear in the Bulletin of the Malaysian Mathematical Sciences Society. Paper submitted 12-Feb-2013; revised 07-May-2013; accepted for publication 05Jun-2013. 
The text is organized as follows. In Section 2 we present some preliminary results and basic definitions necessary in the sequel. Namely, we briefly present the nabla and the delta calculus 3. 4. We also recall the notions of diamond- $\alpha$ derivative and integral, as a linear combination of the delta and nabla derivatives and integral, respectively [5, 8, 9, In Section 3 we introduce our diamond integral, derive some of its properties, and prove some integral inequalities. We end with Section 4 of conclusion.

\section{Preliminaries}

A nonempty closed subset of $\mathbb{R}$ is called a time scale and is denoted by $\mathbb{T}$. We assume that a time scale has the topology inherited from $\mathbb{R}$ with the standard topology. Two jump operators are considered: the forward jump operator $\sigma: \mathbb{T} \rightarrow \mathbb{T}$, defined by $\sigma(t):=\inf \{s \in \mathbb{T}: s>t\}$ with $\inf \emptyset=\sup \mathbb{T}$ (i.e., $\sigma(M)=M$ if $\mathbb{T}$ has a maximum $M$ ), and the backward jump operator $\rho: \mathbb{T} \rightarrow \mathbb{T}$ defined by $\rho(t):=\sup \{s \in \mathbb{T}: s<t\}$ with $\sup \emptyset=\inf \mathbb{T}$ (i.e., $\rho(m)=m$ if $\mathbb{T}$ has a minimum $m$ ). If $\sup \mathbb{T}$ is finite and left-scattered, then we define $\mathbb{T}^{\kappa}:=\mathbb{T} \backslash\{\sup \mathbb{T}\}$, otherwise $\mathbb{T}^{\kappa}:=\mathbb{T}$; if inf $\mathbb{T}$ is finite and right-scattered, then $\mathbb{T}_{\kappa}:=\mathbb{T} \backslash\{\inf \mathbb{T}\}$, otherwise $\mathbb{T}_{\kappa}:=\mathbb{T}$. We set $\mathbb{T}_{\kappa}^{\kappa}:=\mathbb{T}_{\kappa} \cap \mathbb{T}^{\kappa}$.

Definition $1([3])$. We say that a function $f: \mathbb{T} \rightarrow \mathbb{R}$ is delta differentiable at $t \in \mathbb{T}^{\kappa}$ if there exists a number $f^{\Delta}(t)$ such that, for all $\varepsilon>0$, there exists a neighborhood $U$ of $t$ such that

$$
\left|f(\sigma(t))-f(s)-f^{\Delta}(t)(\sigma(t)-s)\right| \leqslant \varepsilon|\sigma(t)-s|
$$

for all $s \in U$. We call $f^{\Delta}(t)$ the delta derivative of $f$ at $t$ and we say that $f$ is delta differentiable if $f$ is delta differentiable for all $t \in \mathbb{T}^{\kappa}$.

Definition $2(\underline{3})$. We say that a function $f: \mathbb{T} \rightarrow \mathbb{R}$ is nabla differentiable at $t \in \mathbb{T}_{\kappa}$ if there exists a number $f^{\nabla}(t)$ such that, for all $\varepsilon>0$, there exists a neighborhood $V$ of $t$ such that

$$
\left|f(\rho(t))-f(s)-f^{\nabla}(t)(\rho(t)-s)\right| \leqslant \varepsilon|\rho(t)-s|
$$

for all $s \in V$. We call $f^{\nabla}(t)$ the nabla derivative of $f$ at $t$ and we say that $f$ is nabla differentiable if $f$ is nabla differentiable for all $t \in \mathbb{T}_{\kappa}$.

Remark 1. If $\mathbb{T}=\mathbb{R}$, then $f^{\Delta}=f^{\nabla}=f^{\prime}$, where $f^{\prime}$ denotes the usual derivative on $\mathbb{R}$. If $\mathbb{T}=\mathbb{Z}$, then $f^{\Delta}(t)=f(t+1)-f(t)$ and $f^{\nabla}(t)=f(t)-f(t-1)$, i.e., $f^{\Delta}$ and $f^{\nabla}$ are, respectively, the usual forward and backward difference operators. For any time scale $\mathbb{T}$, if $f$ is a constant function, then $f^{\Delta}=f^{\nabla} \equiv 0$; if $f(t)=k t$ for some constant $k$, then $f^{\Delta}=f^{\nabla} \equiv k$.

Let $a, b \in \mathbb{T}, a<b$. In what follows we denote $[a, b]_{\mathbb{T}}:=\{t \in \mathbb{T}: a \leq t \leq b\}$.

Definition $3([3])$. A function $F: \mathbb{T} \rightarrow \mathbb{R}$ is said to be a delta antiderivative of $f: \mathbb{T} \rightarrow \mathbb{R}$, provided $F^{\Delta}(t)=f(t)$ for all $t \in \mathbb{T}^{\kappa}$. For all $a, b \in \mathbb{T}, a<b$, the delta integral of $f$ from $a$ to $b$ (or on $[a, b]_{\mathbb{T}}$ ) is defined by

$$
\int_{a}^{b} f(t) \Delta t=F(b)-F(a) .
$$

Definition 4 ([3]). A function $G: \mathbb{T} \rightarrow \mathbb{R}$ is said to be a nabla antiderivative of $g: \mathbb{T} \rightarrow \mathbb{R}$, provided $G^{\nabla}(t)=g(t)$ for all $t \in \mathbb{T}_{k}$. For all $a, b \in \mathbb{T}, a<b$, the nabla integral of $g$ from $a$ to $b$ (or on $[a, b]_{\mathbb{T}}$ ) is defined by

$$
\int_{a}^{b} g(t) \nabla t=G(b)-G(a) .
$$

For the properties of the delta and nabla integrals we refer the readers to [3, 4]. 
Remark 2. If $\mathbb{T}=\mathbb{R}$, then $\int_{a}^{b} f(t) \Delta t=\int_{a}^{b} f(t) \nabla t=\int_{a}^{b} f(t) d t$, where the last integral is the usual Riemman integral. If $\mathbb{T}=h \mathbb{Z}$, for some $h>0$, and $a, b \in \mathbb{T}, a<b$, then $\int_{a}^{b} f(t) \Delta t=$ $\sum_{k=\frac{a}{h}}^{\frac{b}{h}-1} h f(k h)$ and $\int_{a}^{b} f(t) \nabla t=\sum_{k=\frac{a}{h}+1}^{\frac{b}{h}} h f(k h)$.

To provide a shorthand notation, for a function $f: \mathbb{T} \rightarrow \mathbb{R}$ we let $f^{\sigma}(t):=f(\sigma(t))$ and $f^{\rho}(t):=f(\rho(t))$.

Definition $5\left([8)\right.$. Let $t, s \in \mathbb{T}$ and define $\mu_{t s}:=\sigma(t)-s$ and $\eta_{t s}:=\rho(t)-s$. We say that a function $f: \mathbb{T} \rightarrow \mathbb{R}$ is diamond- $\alpha$ differentiable at $t \in \mathbb{T}_{\kappa}^{\kappa}$ if there exists a number $f^{\diamond_{\alpha}}(t)$ such that, for all $\varepsilon>0$, there exists a neighborhood $U$ of $t$ such that, for all $s \in U$,

$$
\left|\alpha\left[f^{\sigma}(t)-f(s)\right] \eta_{t s}+(1-\alpha)\left[f^{\rho}(t)-f(s)\right] \mu_{t s}-f^{\diamond_{\alpha}}(t) \mu_{t s} \eta_{t s}\right| \leqslant \varepsilon\left|\mu_{t s} \eta_{t s}\right| .
$$

A function $f$ is said to be diamond- $\alpha$ differentiable provided $f^{\diamond_{\alpha}}(t)$ exists for all $t \in \mathbb{T}_{\kappa}^{\kappa}$.

Theorem 1 ([8]). Let $0 \leqslant \alpha \leqslant 1$ and let $f$ be both nabla and delta differentiable at $t \in \mathbb{T}_{\kappa}^{\kappa}$. Then $f$ is diamond- $\alpha$ differentiable at $t$ and

$$
f^{\diamond_{\alpha}}(t)=\alpha f^{\Delta}(t)+(1-\alpha) f^{\nabla}(t) .
$$

Remark 3. If $\alpha=1$, then the diamond- $\alpha$ derivative reduces to the delta derivative; if $\alpha=0$, then the diamond- $\alpha$ derivative coincides with the nabla derivative.

Remark 4 . The equality (10) is given as the definition of the diamond- $\alpha$ derivative in [5].

Definition 6 ([8]). Let $a, b \in \mathbb{T}, a<b, h: \mathbb{T} \rightarrow \mathbb{R}$ and $\alpha \in[0,1]$. The diamond- $\alpha$ integral (or $\diamond_{\alpha}$-integral) of $h$ from $a$ to $b$ (or on $[a, b]_{\mathbb{T}}$ ) is defined by

$$
\int_{a}^{b} h(t) \diamond_{\alpha} t=\alpha \int_{a}^{b} h(t) \Delta t+(1-\alpha) \int_{a}^{b} h(t) \nabla t,
$$

provided $h$ is delta and nabla integrable on $[a, b]_{\mathbb{T}}$.

For properties, results, and integral inequalities concerning the diamond- $\alpha$ integral, we refer the reader to [10, 11, 12, 13] and references therein.

\section{The diamond integral}

In the classical calculus, one can find several attempts to define a symmetric integral - see, e.g., [7, 14. However, those integrals invert only "approximately" the symmetric derivatives [14]. In the quantum setting, symmetric integrals are available, namely the $q$-symmetric integral - see, e.g., [15, 16] - and the Hahn symmetric integral, that inverts the Hahn symmetric derivative [17]. In the more general setting of time scales, the problem of determining a symmetric integral is an interesting open question. In particular, such integral would be new, and of great interest, even in the classical case $\mathbb{T}=\mathbb{R}$. Given the similarities and the advantages of the recent symmetric derivative with respect to the diamond- $\alpha$ derivative [6], we claim that the diamond integral here introduced bring us closer to the construction of a genuine symmetric integral on time scales.

We borrow from [6] the real function

$$
\gamma(t):=\lim _{s \rightarrow t} \frac{\sigma(t)-s}{\sigma(t)+2 t-2 s-\rho(t)},
$$

which plays an important role in the definition of our diamond integral (Definition 7). Note that $\gamma$ is well defined, $0 \leqslant \gamma(t) \leqslant 1$ for all $t \in \mathbb{T}$, and

$$
\gamma(t)= \begin{cases}\frac{1}{2} & \text { if } t \text { is dense, } \\ \frac{\sigma(t)-t}{\sigma(t)-\rho(t)} & \text { if } t \text { is not dense. }\end{cases}
$$


Definition 7 (diamond integral). Let $f: \mathbb{T} \rightarrow \mathbb{R}$ and $a, b \in \mathbb{T}, a<b$. The diamond integral (or $\diamond$-integral) of $f$ from $a$ to $b$ (or on $[a, b]_{\mathbb{T}}$ ) is given by

$$
\int_{a}^{b} f(t) \diamond t:=\int_{a}^{b} \gamma(t) f(t) \Delta t+\int_{a}^{b}(1-\gamma(t)) f(t) \nabla t
$$

provided $\gamma f$ is delta integrable and $(1-\gamma) f$ is nabla integrable on $[a, b]_{\mathbb{T}}$. We say that the function $f$ is diamond integrable (or $\diamond$ - integrable) on $[a, b]_{\mathbb{T}}$ if it is $\diamond$-integrable for all $a, b \in \mathbb{T}$.

Remark 5. The $\diamond$-integral coincides with the $\diamond_{\alpha}$-integral when the function $\gamma$ is constant and equal to $\alpha$. There are several important time scales where this happens. For instance, when $\mathbb{T}=\mathbb{R}$ or $\mathbb{T}=h \mathbb{Z}, h>0$, the $\diamond$-integral is equal to the $\diamond_{\frac{1}{2}}$-integral. Since the fundamental theorem of calculus is not valid for the $\nabla_{\alpha}$-integral (see [5]), it is clear that the fundamental theorem of calculus is also not valid for the $\diamond$-integral. Hence, the diamond integral is not a genuine symmetric integral on time scales. Despite this limitation, we show that the new integral satisfies some important properties.

Example 1. Let $f: \mathbb{Z} \rightarrow \mathbb{R}$ be defined by $f(t)=t^{2}$. Then,

$$
\int_{0}^{2} f(t) \diamond t=\frac{1}{2} \int_{0}^{2} f(t) \Delta t+\frac{1}{2} \int_{0}^{2} f(t) \nabla t=\frac{1}{2} \sum_{t=0}^{1} f(t)+\frac{1}{2} \sum_{t=1}^{2} f(t)=\frac{1}{2}(0+1)+\frac{1}{2}(1+4)=3 .
$$

Example 2. Let $f:[0,1] \cup\{2,4\} \rightarrow \mathbb{R}$ be defined by $f(t)=1$. Then,

$$
\begin{aligned}
\int_{0}^{4} f(t) \diamond t & =\int_{0}^{1} 1 d t+\int_{1}^{2} \gamma(t) \Delta t+\int_{2}^{4} \gamma(t) \Delta t+\int_{1}^{2}(1-\gamma(t)) \nabla t+\int_{2}^{4}(1-\gamma(t)) \nabla t \\
& =1+\gamma(1)+2 \cdot \gamma(2)+(1-\gamma(2))+2 \cdot(1-\gamma(4))=\frac{17}{3} .
\end{aligned}
$$

Note that, in this example, and independently of the value of $\alpha$, our diamond integral is different from the diamond- $\alpha$ integral:

$$
\int_{0}^{4} f(t) \diamond_{\alpha} t=\int_{0}^{1} f(t) \diamond t+\alpha \int_{1}^{4} 1 \Delta t+(1-\alpha) \int_{1}^{4} 1 \nabla t=1+3 \alpha+(1-\alpha) 3=4 \neq \frac{17}{3} .
$$

The $\diamond$-integral has the following properties.

Theorem 2. Let $f, g: \mathbb{T} \rightarrow \mathbb{R}$ be $\diamond$-integrable on $[a, b]_{\mathbb{T}}$. Let $c \in[a, b]_{\mathbb{T}}$ and $\lambda \in \mathbb{R}$. Then,

1. $\int_{a}^{a} f(t) \diamond t=0$;

2. $\int_{a}^{b} f(t) \diamond t=\int_{a}^{c} f(t) \diamond t+\int_{c}^{b} f(t) \diamond t$;

3. $\int_{a}^{b} f(t) \diamond t=-\int_{b}^{a} f(t) \diamond t$;

4. $f+g$ is $\diamond$-integrable on $[a, b]_{\mathbb{T}}$ and $\int_{a}^{b}(f+g)(t) \diamond t=\int_{a}^{b} f(t) \diamond t+\int_{a}^{b} g(t) \diamond t$;

5. $\lambda f$ is $\diamond$-integrable on $[a, b]_{\mathbb{T}}$ and $\int_{a}^{b} \lambda f(t) \diamond t=\lambda \int_{a}^{b} f(t) \diamond t$;

6. $f g$ is $\diamond$-integrable on $[a, b]_{\mathbb{T}}$;

7. for $p>0,|f|^{p}$ is $\diamond$-integrable on $[a, b]_{\mathbb{T}}$; 
8. if $f(t) \leqslant g(t)$ for all $t \in[a, b]_{\mathbb{T}}$, then $\int_{a}^{b} f(t) \diamond t \leqslant \int_{a}^{b} g(t) \diamond t$;

9. $|f|$ is $\diamond$-integrable on $[a, b]_{\mathbb{T}}$ and $\left|\int_{a}^{b} f(t) \diamond t\right| \leqslant \int_{a}^{b}|f(t)| \diamond t$.

Proof. The results follow straightforwardly from the analogous properties of the nabla and delta integrals.

Next we extend to the diamond integral some results obtained in [10, 11] for the $\diamond_{\alpha}$-integral.

Theorem 3 (Mean value theorem for the diamond integral). Let $f, g: \mathbb{T} \rightarrow \mathbb{R}$ be bounded and $\diamond$-integrable functions on $[a, b]_{\mathbb{T}}$, and let $g$ be nonnegative or nonpositive on $[a, b]_{\mathbb{T}}$. Let $m$ and $M$ be the infimum and supremum, respectively, of function $f$. Then, there exists a real number $K$ satisfying the inequalities $m \leqslant K \leqslant M$ such that

$$
\int_{a}^{b}(f g)(t) \diamond t=K \int_{a}^{b} g(t) \diamond t .
$$

Proof. Without loss of generality, we suppose that $g$ is nonnegative on $[a, b]_{\mathbb{T}}$. Since, for all $t \in[a, b]_{\mathbb{T}}, m \leqslant f(t) \leqslant M$ and $g(t) \geqslant 0$, then $m g(t) \leqslant f(t) g(t) \leqslant M g(t)$ for all $t \in[a, b]_{\mathbb{T}}$. Each of the functions $m g, f g$ and $M g$ is $\diamond$-integrable from $a$ to $b$ and, by Theorem 2 , one has

$$
m \int_{a}^{b} g(t) \diamond t \leqslant \int_{a}^{b} f(t) g(t) \diamond t \leqslant M \int_{a}^{b} g(t) \diamond t .
$$

If $\int_{a}^{b} g(t) \diamond t=0$, then $\int_{a}^{b} f(t) g(t) \diamond t=0$ and we can choose any $K \in[m, M]$. If $\int_{a}^{b} g(t) \diamond t>0$, then $m \leqslant \frac{\int_{a}^{b} f(t) g(t) \diamond t}{\int_{a}^{b} g(t) \diamond t} \leqslant M$, and we choose $K:=\frac{\int_{a}^{b} f(t) g(t) \diamond t}{\int_{a}^{b} g(t) \diamond t}$.

We now present $\diamond$-versions of Hölder's, Cauchy-Schwarz's and Minkowski's inequalities.

Theorem 4 (Hölder's inequality for the diamond integral). If $f, g: \mathbb{T} \rightarrow \mathbb{R}$ are $\diamond$-integrable on $[a, b]_{\mathbb{T}}$, then

$$
\int_{a}^{b}|f(t) g(t)| \diamond t \leqslant\left(\int_{a}^{b}|f(t)|^{p} \diamond t\right)^{\frac{1}{p}}\left(\int_{a}^{b}|g(t)|^{q} \diamond t\right)^{\frac{1}{q}},
$$

where $p>1$ and $q=\frac{p}{p-1}$.

Proof. For $\lambda, \beta \in \mathbb{R}_{0}^{+}$and $p, q$ such that $p>1$ and $\frac{1}{p}+\frac{1}{q}=1$, the following inequality holds (Young's inequality):

$$
\lambda^{\frac{1}{p}} \beta^{\frac{1}{q}} \leqslant \frac{\lambda}{p}+\frac{\beta}{q}
$$

Without loss of generality, let us suppose that $\left(\int_{a}^{b}|f(t)|^{p} \diamond t\right)\left(\int_{a}^{b}|g(t)|^{q} \diamond t\right) \neq 0$ (note that both integrals exist by Theorem 2). Define

$$
\lambda(t):=\frac{|f(t)|^{p}}{\int_{a}^{b}|f(\tau)|^{p} \diamond \tau} \text { and } \beta(t):=\frac{|g(t)|^{q}}{\int_{a}^{b}|g(\tau)|^{q} \diamond \tau} .
$$


Since both functions $\lambda$ and $\beta$ are $\diamond$-integrable on $[a, b]_{\mathbb{T}}$, then

$$
\begin{aligned}
& \int_{a}^{b} \frac{|f(t)|}{\left(\int_{a}^{b}|f(\tau)|^{p} \diamond \tau\right)^{\frac{1}{p}}} \frac{|g(t)|}{\left(\int_{a}^{b}|g(\tau)|^{q} \diamond \tau\right)^{\frac{1}{q}}} \diamond t \\
& \quad=\int_{a}^{b}(\lambda(t))^{\frac{1}{p}}(\beta(t))^{\frac{1}{q}} \diamond t \\
& \quad \leqslant \int_{a}^{b}\left(\frac{\lambda(t)}{p}+\frac{\beta(t)}{q}\right) \diamond t \\
& \quad=\frac{1}{p} \int_{a}^{b}\left(\frac{|f(t)|^{p}}{\int_{a}^{b}|f(\tau)|^{p} \diamond \tau}\right) \diamond t+\frac{1}{q} \int_{a}^{b}\left(\frac{|g(t)|^{q}}{\int_{a}^{b}|g(\tau)|^{q} \diamond \tau}\right) \diamond t=\frac{1}{p}+\frac{1}{q}=1,
\end{aligned}
$$

proving the intended result.

Corollary 1 (Cauchy-Schwarz's inequality for the diamond integral). If $f, g: \mathbb{T} \rightarrow \mathbb{R}$ are $\diamond$ integrable on $[a, b]_{\mathbb{T}}$, then

$$
\int_{a}^{b}|f(t) g(t)| \diamond t \leqslant \sqrt{\left(\int_{a}^{b}|f(t)|^{2} \diamond t\right)\left(\int_{a}^{b}|g(t)|^{2} \diamond t\right)} .
$$

Proof. This is a particular case of Theorem 4 where $p=2=q$.

Theorem 5 (Minkowski's inequality for the diamond integral). If $f, g: \mathbb{T} \rightarrow \mathbb{R}$ is $\diamond$-integrable on $[a, b]_{\mathbb{T}}$ and $p>1$, then

$$
\left(\int_{a}^{b}|f(t)+g(t)|^{p} \diamond t\right)^{\frac{1}{p}} \leqslant\left(\int_{a}^{b}|f(t)|^{p} \diamond t\right)^{\frac{1}{p}}+\left(\int_{a}^{b}|g(t)|^{p} \diamond t\right)^{\frac{1}{p}} .
$$

Proof. If $\int_{a}^{b}|f(t)+g(t)|^{p} \diamond t=0$, then the result is trivial. Suppose $\int_{a}^{b}|f(t)+g(t)|^{p} \diamond t \neq 0$. Since

$$
\begin{aligned}
\int_{a}^{b}|f(t)+g(t)|^{p} \diamond t & =\int_{a}^{b}|f(t)+g(t)|^{p-1}|f(t)+g(t)| \diamond t \\
& \leqslant \int_{a}^{b}|f(t)||f(t)+g(t)|^{p-1} \diamond t+\int_{a}^{b}|g(t)||f(t)+g(t)|^{p-1} \diamond t,
\end{aligned}
$$

then, by Hölder's inequality (Theorem 4 ) with $q=\frac{p}{p-1}$, we obtain that

$$
\int_{a}^{b}|f(t)+g(t)|^{p} \diamond t \leqslant\left[\left(\int_{a}^{b}|f(t)|^{p} \diamond t\right)^{\frac{1}{p}}+\left(\int_{a}^{b}|g(t)|^{p} \diamond t\right)^{\frac{1}{p}}\right]\left(\int_{a}^{b}|f(t)+g(t)|^{(p-1) q} \diamond t\right)^{\frac{1}{q}} .
$$

Dividing both sides by $\left(\int_{a}^{b}|f(t)+g(t)|^{p} \diamond t\right)^{\frac{1}{q}}$, we arrive to the intended inequality:

$$
\left(\int_{a}^{b}|f(t)+g(t)|^{p} \diamond t\right)^{\frac{1}{p}} \leqslant\left(\int_{a}^{b}|f(t)|^{p} \diamond t\right)^{\frac{1}{p}}+\left(\int_{a}^{b}|g(t)|^{p} \diamond t\right)^{\frac{1}{p}} .
$$




\section{Conclusion}

Combined delta and nabla derivatives, as well as combined integrals, are increasingly getting more attention in approximating functions and solutions of differential equations. The recent symmetric derivative on time scales [6] unifies the symmetric derivatives of classical analysis and quantum calculus. Moreover, it is a generalization of the delta and nabla derivatives on time scales. It is important to note that the symmetric derivative on time scales is different from the delta and nabla derivatives: for example, the absolute value function is neither delta nor nabla differentiable but it is symmetric differentiable. When a function is simultaneously delta and nabla differentiable, the symmetric derivative can then be written as a time dependent combination of those derivatives. In this paper we introduce a new type of integral on time scales, the diamond integral, based on the definition of the symmetric derivative [6. Instead of a constant parameter, like in the definition of the diamond- $\alpha$ integral [5, 8, we propose a new version of the diamond integral with a time dependent parameter. The diamond integral is the Riemman integral when the time scale is $\mathbb{T}=\mathbb{R}$ and is an arithmetic average of the delta and nabla integrals when the time scale is $\mathbb{T}=\mathbb{Z}$ or any other time scale with the forward graininess function $\mu(t):=\sigma(t)-t$ or the backward graininess function $\nu(t):=t-\rho(t)$ constant. We prove that this new integral satisfies important properties such as the mean value theorem and Hölder and Minkowski type inequalities. It is well known that integral inequalities on time scales play a major role in the development of other areas of mathematics. For example, in the calculus of variations, Holder and Minkowski type inequalities can be used to find explicitly the extremizers for some classes of variational problems on time scales [18. We trust that our diamond integral on time scales is interesting and useful, and will lead to subsequent investigations with important applications.

\section{Acknowledgments}

This work was supported by FEDER funds through COMPETE - Operational Programme Factors of Competitiveness ("Programa Operacional Factores de Competitividade") and by Portuguese funds through the Center for Research and Development in Mathematics and Applications (University of Aveiro) and the Portuguese Foundation for Science and Technology ("FCT - Fundação para a Ciência e a Tecnologia"), within project PEst-C/MAT/UI4106/2011 with COMPETE number FCOMP-01-0124-FEDER-022690. Brito da Cruz was also supported by FCT through the Ph.D. fellowship SFRH/BD/33634/2009. The authors are grateful to two anonymous referees for valuable comments and helpful suggestions.

\section{References}

[1] B. Aulbach and S. Hilger, A unified approach to continuous and discrete dynamics, in Qualitative theory of differential equations (Szeged, 1988), 37-56, Colloq. Math. Soc. János Bolyai, 53 North-Holland, Amsterdam, 1990.

[2] S. Hilger, Analysis on measure chains - a unified approach to continuous and discrete calculus, Results Math. 18 (1990), no. 1-2, 18-56.

[3] M. Bohner and A. Peterson, Dynamic equations on time scales, Birkhäuser Boston, Boston, MA, 2001.

[4] M. Bohner and A. Peterson, Advances in dynamic equations on time scales, Birkhäuser Boston, Boston, MA, 2003.

[5] Q. Sheng, M. Fadag, J. Henderson and J. M. Davis, An exploration of combined dynamic derivatives on time scales and their applications, Nonlinear Anal. Real World Appl. 7 (2006), no. $3,395-413$. 
[6] A. M. C. Brito da Cruz, N. Martins and D. F. M. Torres, Symmetric differentiation on time scales, Appl. Math. Lett. 26 (2013), no. 2, 264-269. arXiv:1209.2094

[7] B. S. Thomson, Symmetric properties of real functions, Monographs and Textbooks in Pure and Applied Mathematics, 183, Dekker, New York, 1994.

[8] J. W. Rogers, Jr. and Q. Sheng, Notes on the diamond- $\alpha$ dynamic derivative on time scales, J. Math. Anal. Appl. 326 (2007), no. 1, 228-241.

[9] Q. Sheng, Hybrid approximations via second order combined dynamic derivatives on time scales, Electron. J. Qual. Theory Differ. Equ. 2007 (2007), no. 17, 13 pp.

[10] A. B. Malinowska and D. F. M. Torres, On the diamond-alpha Riemann integral and mean value theorems on time scales, Dynam. Systems Appl. 18 (2009), no. 3-4, 469-481. arXiv:0804.4420

[11] M. R. Sidi Ammi, R. A. C. Ferreira and D. F. M. Torres, Diamond- $\alpha$ Jensen's inequality on time scales, J. Inequal. Appl. 2008 (2008), Art. ID 576876, 13 pp. arXiv:0712.1680

[12] D. Mozyrska and D. F. M. Torres, Diamond-alpha polynomial series on time scales, Int. J. Math. Stat. 5 (2009), A09, 92-101. arXiv:0805.0274

[13] M. R. Sidi Ammi and D. F. M. Torres, Hölder's and Hardy's two dimensional diamondalpha inequalities on time scales, An. Univ. Craiova Ser. Mat. Inform. 37 (2010), no. 1, 1-11. arXiv:0910.4115

[14] G. E. Cross and B. S. Thomson, Symmetric integrals and trigonometric series, Dissertationes Math. (Rozprawy Mat.) 319 (1992), 49 pp.

[15] V. Kac and P. Cheung, Quantum calculus, Universitext, Springer, New York, 2002.

[16] A. M. C. Brito da Cruz and N. Martins, The $q$-symmetric variational calculus, Comput. Math. Appl. 64 (2012), no. 7, 2241-2250.

[17] A. M. C. Brito da Cruz, N. Martins and D. F. M. Torres, Hahn's symmetric quantum variational calculus, Numer. Algebra Control Optim. 3 (2013), no. 1, 77-94. arXiv:1209.1530

[18] M. J. Bohner, R. A. C. Ferreira and D. F. M. Torres, Integral inequalities and their applications to the calculus of variations on time scales, Math. Inequal. Appl. 13 (2010), no. 3, 511-522. arXiv:1001.3762 